\title{
Scanning Electron Microscopy of Nanoscale Chemical Patterns
}

\author{
Charan Srinivasan, ${ }^{+, \pm}$Thomas J. Mullen, ${ }^{\dagger}$ J. Nathan Hohman, ${ }^{\dagger}$ Mary E. Anderson, ${ }^{\dagger}$ Arrelaine A. Dameron, ${ }^{\dagger, \perp}$ \\ Anne M. Andrews, ${ }^{\S}$ Elizabeth C. Dickey, Mark W. Horn, ${ }^{\neq}$and Paul S. Weiss ${ }^{\dagger, *}$ \\ ${ }^{\dagger}$ Departments of Chemistry and Physics, ${ }^{ \pm}$Department of Engineering Science and Mechanics, ${ }^{5}$ Department of Veterinary and Biomedical Sciences and Huck Institutes of \\ the Life Sciences, and "Department of Materials Science and Engineering, The Pennsylvania State University, University Park, Pennsylvania 16802-6300. ${ }^{\perp}$ Current \\ address: Dynamic Organic Light, 2410 Trade Center Ave., Longmont, C0 80503.
}

M olecular self-assembly has emerged as a key route to create and to manipulate functional surfaces for capabilities that span biospecific recognition to molecular electronics. ${ }^{1-4}$ In particular, fundamental research focusing on alkanethiolate self-assembled monolayers (SAMs) on Au has gained considerable attention due to the spontaneous formation of the versatile Au-S bond, resulting in highly ordered molecular films that can be fabricated over large areas. Key to advances in this field has been the concurrent development of soft-lithographic techniques such as microcontact printing $(\mu \mathrm{CP}),{ }^{5}$ micromolding in capillaries, ${ }^{6}$ and dip-pen nanolithography, ${ }^{7}$ which can be used to control the nanoscopic properties of SAMs using micrometer-scale fabrication techniques.

In $\mu \mathrm{CP}$, for example, a micropatterned stamp coated with the desired ink molecule is brought into conformal contact with a suitable substrate. Consequently, a chemical pattern that replicates the elastomeric stamp geometry is created on the substrate via the transfer of the ink molecules only at the protruding regions of the stamp. Here, we directly imaged chemical patterns generated using $\mu \mathrm{CP}$ and two related techniques-microdisplacement printing $(\mu D P)^{8}$ and microcontact insertion printing ( $\mu \mathrm{ClP}) .{ }^{9}$ We also imaged chemical patterns created using lithography-assisted chemical patterning (LACP). ${ }^{10}$ These patterned surfaces were imaged using a field emission scanning electron microscope (FESEM) and corroborated with measurements using scanning tunneling microscopy (STM) and lateral force microscopy (LFM).

A fundamental limitation of $\mu \mathrm{CP}$ to create chemical patterns is the tendency of

www.acsnano.org
ABSTRACT A series of nanoscale chemical patterning methods based on soft and hybrid nanolithographies have been characterized using scanning electron microscopy with corroborating evidence from scanning tunneling microscopy and lateral force microscopy. We demonstrate and discuss the unique advantages of the scanning electron microscope as an analytical tool to image chemical patterns of molecules highly diluted within a host selfassembled monolayer and to distinguish regions of differential mass coverage in patterned self-assembled monolayers. We show that the relative contrast of self-assembled monolayer patterns in scanning electron micrographs depends on the operating primary electron beam voltage, monolayer composition, and monolayer order, suggesting that secondary electron emission and scattering can be used to elucidate chemical patterns.

KEYWORDS: chemical patterning · nanolithography · scanning electron microscopy $\cdot$ self-assembly $\cdot$ soft-lithography $\cdot$ microcontact printing

low-molecular-weight molecules to diffuse laterally and spread across the substrate surface. This results in poor pattern fidelity and, in some cases, even pattern dissolution..$^{8,11}$ By modifying and tailoring the intermolecular interactions of a preexisting SAM, we have developed novel softlithographic techniques that broaden the scope of $\mu \mathrm{CP}$. Microdisplacement printing utilizes a preassembled, labile SAM of 1-adamantanethiol (AD) on the surface to improve the fidelity of chemical patterns created by contact printing and permits patterning of low-molecular-weight molecules. ${ }^{8}$ Microcontact insertion printing is a technique for the creation of micrometersize chemical patterns of isolated, inserted molecules with dilute placement and low coverages $(\sim 0.5-15 \%)$ in controlled matrices. ${ }^{9}$ Although $\mu \mathrm{CP}$ is a ubiquitous method for benchtop fabrication of chemical patterns, it has been embraced only to a limited extent by industry because of the process variability and the lack of suitable metrology tools.

In contrast, photolithography is a wellcharacterized, mature technology widely used in semiconductor manufacturing.
*Address correspondence to
stm@psu.edu.

Received for review June 29, 2007 and accepted September 24, 2007.

10.1021/nn7000799 CCC: $\$ 37.00$

(c) XXXX American Chemical Society 


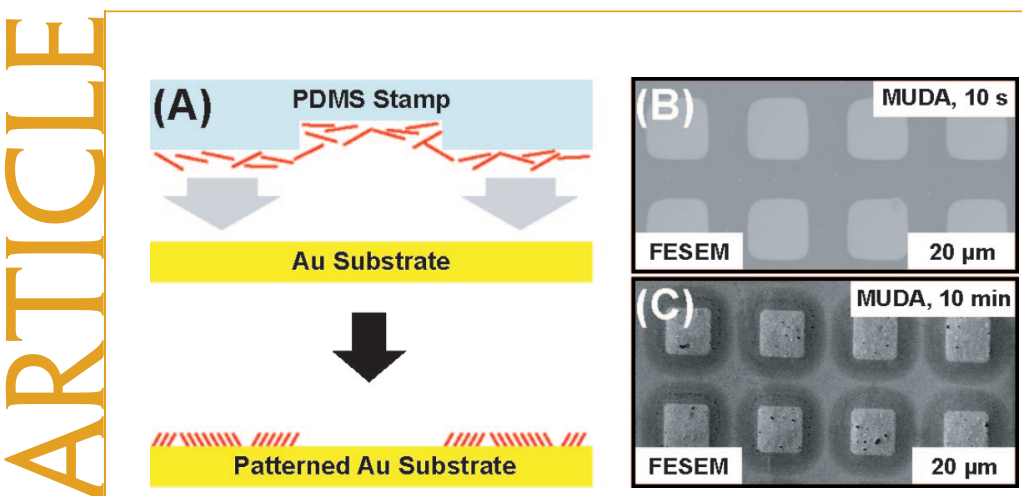

Figure 1. (A) Schematic (not to scale) depicting microcontact ( $\mu \mathrm{CP}$ ) printing with an 11-mercaptoundecanoic acid (MUDA, red lines)-inked stamp. The molecularly inked stamp is contacted directly onto a Au\{111\} substrate, resulting in patterned regions of MUDA that mirror the relief pattern on the stamp. $(B, C)$ Field emission scanning electron microscopy (FESEM) images of patterned Au\{111\} fabricated by $\mu \mathrm{CP}$ using $10 \mu \mathrm{m} \times 10 \mu \mathrm{m}$ posts on a poly(dimethylsiloxane) stamp inked with a $25 \mathrm{mM}$ MUDA solution. The stamp-substrate contact times were (B) $10 \mathrm{~s}$ and (C) $10 \mathrm{~min}$. The regions with higher intensity correspond to the stamped MUDA, and the lower intensity background is the $\mathrm{Au}\{111\}$ substrate. Microcontact printing for $10 \mathrm{~s}$ results in better pattern fidelity but poorer molecular ordering of the SAM. Microcontact printing for $10 \mathrm{~min}$ improved the molecular ordering of the $S A M,{ }^{11,19,26}$ but the pattern contained diffuse regions that surrounded the square-shaped regions of the intended chemical patterns. These surrounding areas of lower coverage form due to lateral surface diffusion of the MUDA molecules, which have low intermolecular interactions. These micrographs were acquired at a primary electron beam voltage of $1 \mathrm{kV}$ and a collector voltage of $+300 \mathrm{~V}$.

Lithography-assisted chemical patterning utilizes a robust lithographic resist that is capable of withstanding self-assembly deposition conditions and is patterned by photolithography to create high-quality chemical patterns. ${ }^{10}$

In previous work, chemical patterns of SAMs and Langmuir-Blodgett films were imaged with a SEM. ${ }^{12-14}$ Both experimental and theoretical ${ }^{15}$ considerations were applied to rationalize the resulting relative intensities observed in the micrographs. The contrast observed in scanning electron micrographs of chemical patterns is due to spatial differences in surface work function and secondary electron emission between the different molecular components. These, in turn, were found to be modulated differently on the basis of the terminal functionality, alkyl chain length, and electroncloud density of the SAMs, the thicknesses of adsorbed organic overlayers on SAMs, and the molecular ordering of the Langmuir-Blodgett films. For example, the SEM intensity decreases with decreasing alkyl chain length in SAMs, with increasing thicknesses of organic overlayers adsorbed over SAMs, and with poorer molecular ordering in Langmuir-Blodgett films. ${ }^{12-14}$ In this article, we show that the SEM is also capable of nondestructively imaging very dilute (down to $\sim 0.5 \%$ ) fractional coverages of patterned, isolated molecules within a host matrix in $\mu \mathrm{CIP}$ and also the diffused regions of chemical patterns created by $\mu \mathrm{CP}$ in a more facile manner than alternative techniques. Furthermore, we validate the qualitative SEM studies with complementary, quantitative information from STM and LFM. Finally, we demonstrate the well-known phenomenon of contrast dependence on operating voltage of the $\mathrm{SEM}_{1}{ }^{16}$ but here we utilize it to image chemical patterns of SAMs.

It is advantageous to develop the SEM as a characterization tool for chemical patterning for the following reasons. First, the SEM is the foremost metrology tool (along with scatterometry) available in semiconductor manufacturing. ${ }^{17}$ Specialized tools, called critical dimension SEMs (CD-SEMs), are available from many commercial vendors. Technologies based on chemical patterning may then be well served by the development of SEM characterization, which is both rapid and capable of large-area imaging. Competing methods such as scanning ellipsometry and atomic force microscopy provide limited sample throughput. Second, the SEM is a relatively non-destructive technique and is highly sensitive to even small variations in surface composition and molecular ordering in patterned SAMs. These aspects render the SEM a vital analytical tool in the development and optimization of various patterning techniques and will help facilitate additional fundamental research and development in this area.

\section{RESULTS AND DISCUSSION}

Patterning and Measurements. Microcontact Printing. In $\mu \mathrm{CP}$, a patterned stamp is used to produce chemical patterns on surfaces that replicate the stamp geometry (Figure 1A). This technique has been the focus of much research, mainly due to the ease of implementation, low capital investment, and ability to pattern large areas $\left(>1 \mathrm{~cm}^{2}\right)$. The fidelity of chemical patterns created by $\mu \mathrm{CP}$ varies with many parameters, including stamp deformation, ${ }^{18}$ back-pressure applied to ensure conformal contact between stamp and substrate, ${ }^{19}$ ink molecule, ${ }^{20}$ ink molecule concentration, ${ }^{20,21}$ and stamping time. ${ }^{11}$ These parameters, in turn, influence the molecular ordering, average packing density, and extent of lateral diffusion across the substrate surface of the patterned SAM. ${ }^{22}$

Here, we employed a SEM to image the regions of differential packing density in a patterned SAM. We chose to restrict the scope of our study to the effect of stamping time, in part because of its considerable importance on both the molecular ordering of the resulting SAM and pattern fidelity. Separate patterned poly(dimethylsiloxane) (PDMS) stamps with $10 \mu \mathrm{m} \times 10 \mu \mathrm{m}$ posts were inked with $25 \mathrm{mM}$ 11-mercaptoundecanoic acid (MUDA) and stamped for $10 \mathrm{~s}$ or $10 \mathrm{~min}$. Subsequently, the patterned substrates were imaged with a FESEM (Figure 1B,C). The regions containing the ink molecules appeared with higher secondary electron intensity when compared to the Au background. Further important aspects of these images are the "halo" diffuse regions that typically surrounded the square-shaped regions of the intended chemical patterns that were printed for longer durations (10 min, Figure 1C). These regions correspond to the ink molecules that diffused laterally and have lower mass coverage compared to 
the patterned $10 \mu \mathrm{m} \times 10 \mu \mathrm{m}$ areas. ${ }^{23}$ The low intermolecular interactions between MUDA molecules increased their susceptibility to spread across the substrate. Similar trends were observed for $\mu \mathrm{CP}$ of 16-mercaptohexadecanoic acid (MHDA), 1-octanethiol (C8), and 1-octadecanethiol (C18) (data not shown) and were consistent with previous reports of increasing lateral surface diffusion rate with decreasing alkyl chain length. ${ }^{11,24-26}$

In related work, Bitterman et al. imaged LangmuirBlodgett film domains with varying molecular packing density and found that regions with higher packing density emit more secondary electrons (i.e., have a higher intensity in scanning electron micrographs). ${ }^{13}$ On the basis of these observations, we conclude that the halo diffuse regions correspond to MUDA SAMs with lower mass coverage and molecular ordering, due to which they are highly defect-ridden. These defects, in turn, interact with and attenuate the secondary electron signal from the Au beneath, resulting in the lower intensity of secondary electron emission, even when compared to the Au background. Such halos have previously been observed; see, for example, ref 9 . The diffuse regions were directly imaged here, as shown in Figure $1 C$, presumably due to the imaging conditions used. We used a primary electron beam with energies ( $1 \mathrm{keV}$ ) much smaller than those used in most previous work, resulting in smaller penetration depths, rendering the scanning electron micrographs more sensitive to small variations in surface composition and structure.

Surface diffusion of the ink molecules can be mitigated by reducing their concentration. However, concentrations below $10 \mathrm{mM}$ resulted in a relatively disordered and liquid-like patterned SAM. ${ }^{20}$ Another parameter that can be leveraged for reducing surface diffusion is the stamping time; shorter stamping times also result in less surface diffusion. However, previously reported spectroscopic measurements showed that printing times from $1 \mathrm{~min}$ to $1 \mathrm{~h}$ are required to eliminate conformational defects (and likely low mass coverage phases) in printed SAMs. ${ }^{11,19,26}$ These studies and the inferences drawn from Figure 1B,C allude to a tradeoff between improving the molecular ordering of the SAM and pattern dissolution upon increasing the stamping time. As discussed in the next section, $\mu \mathrm{DP}$ improves this trade-off by utilizing a preassembled SAM that is sufficiently labile to be displaced by competitive adsorption and is yet well-ordered enough to impede surface diffusion of the ink molecules.

Microdisplacement Printing. Enhancing soft-lithographic microcontact patterning, $\mu \mathrm{DP}$ utilizes a preassembled SAM of AD that is sufficiently labile to be displaced by ink molecules from the raised regions of elastomeric stamps but has sufficient intermolecular interactions to limit the surface diffusion of the adsorbed molecules during and after $\mu \mathrm{CP}$. We have previously reported on the ability of $\mathbf{A D}$ to form well-ordered hexagonally
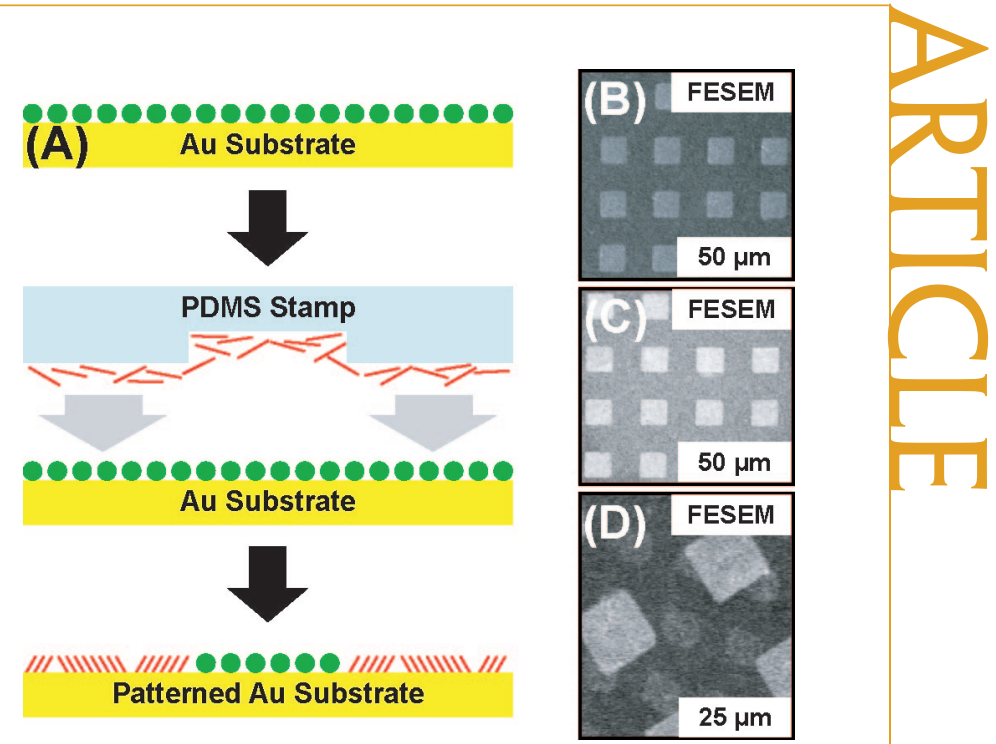

Figure 2. (A) Schematic (not to scale) depicting microdisplacement printing $(\mu D P)$ on a 1-adamantanethiolate (AD, green circles) self-assembled monolayer (SAM) with a poly(dimethylsiloxane) (PDMS) stamp carrying ink molecules (red lines). The molecularly inked stamp is contacted directly onto the AD SAM, resulting in patterned regions of the ink molecule that mirror the relief pattern on the stamp. (B) Field emission scanning electron microscopy (FESEM) image of patterned $A u\{111\}$ fabricated by $\mu \mathrm{DP}$ using $10 \mu \mathrm{m} \times 10 \mu \mathrm{m}$ posts on a PDMS stamp inked with a $25 \mathrm{mM} 11$-mercaptoundecanoic acid solution (MUDA, 10 min stamp-substrate contact time). (C) FESEM image of patterned Au\{111\} fabricated by $\mu$ DP using $10 \mu \mathrm{m} \times 10 \mu \mathrm{m}$ posts on a PDMS stamp inked with a $25 \mathrm{mM}$ 1-dodecanethiol solution (C12, $15 \mathrm{~min}$ stamp-substrate contact time). (D) FESEM image of patterned Au\{111\} fabricated by iterative $\mu D P$ using $10 \mu \mathrm{m} \times 10 \mu \mathrm{m}$ posts on a PDMS stamp inked with $25 \mathrm{mM}$ MUDA and $5 \mu \mathrm{m}$ $\times 5 \mu \mathrm{m}$ posts on a PDMS stamp inked with $25 \mathrm{mM}$ C12 (10 min stamp-substrate contact time for each). The regions of the ink molecules show higher intensity compared to the AD background and better pattern fidelity than $\mu \mathrm{CP}$ for similar stamp-substrate contact times and ink concentrations. These micrographs were acquired at a primary electron beam voltage of $1 \mathrm{kV}$ and a collector voltage of $+300 \mathrm{~V}$.

close-packed SAMs on Au\{111\}, which have larger lattice spacings than $n$-alkanethiolate SAMs. ${ }^{27}$ The AD molecules interact weakly with each other and have a lower molecule-substrate bond density; thus, they are displaced by molecules that interact more strongly and have higher molecule-substrate bond densities to create patterned SAMs. ${ }^{28-31}$ This has been exploited to pattern low-molecular-weight molecules that would otherwise diffuse laterally both during and after patterning. ${ }^{8}$

Shown in Figure $2 A$ is a schematic of $\mu D P$. Selfassembled monolayers of AD were assembled via solution deposition by immersing Au\{111\} substrates in a $10 \mathrm{mM}$ solution of AD for $24 \mathrm{~h}$. Next, a patterned PDMS stamp with $10 \mu \mathrm{m} \times 10 \mu \mathrm{m}$ posts was inked with $25 \mathrm{mM}$ MUDA, stamped for $10 \mathrm{~min}$, and subsequently imaged with a FESEM (Figure 2B). The higher intensity squares correspond to the regions stamped with MUDA; the lower intensity background corresponds to the regions with the unaltered AD SAM. The resulting chemical pattern did not broaden, in contrast to the $\mu C P$ pattern (Figure 1C), for identical molecular ink composition, ink concentration, and stamping time. The remaining AD SAM prevents the lateral spreading of stamped molecules on the substrate surface both 
during and after the stamping process (the images shown here were collected $48 \mathrm{~h}$ after the printing step). Similarly, $10 \mu \mathrm{m} \times 10 \mu \mathrm{m} \mu \mathrm{DP}$ patterns of $25 \mathrm{mM}$ 1-dodecanethiol (C12) stamped for 15 min were created with excellent pattern fidelity (Figure $2 \mathrm{C}$ ). The iterative nature of $\mu \mathrm{DP}$ is demonstrated in Figure 2D. A preformed AD SAM was successively printed for $10 \mathrm{~min}$ each with $25 \mathrm{mM}$ MUDA $(10 \mu \mathrm{m} \times 10 \mu \mathrm{m}$ posts on a PDMS stamp) and $25 \mathrm{mM}$ C12 $(5 \mu \mathrm{m} \times 5 \mu \mathrm{m}$ posts on a PDMS stamp). As noted previously, both of these lowmolecular-weight molecules are difficult to pattern by $\mu C P$ due to their susceptibility to lateral diffusion and thus pattern dissolution. These images show that the preexisting AD SAM is displaced by competitive adsorption only in areas where the elastomeric stamp and substrate were in contact.

Time-dependent STM studies of $\mu$ DP were performed to elucidate the patterning mechanism of $\mu \mathrm{DP}$ with molecular resolution and corroborate the contrast observed in the scanning electron micrographs. ${ }^{8}$ Carboxylic-acid-terminated molecules are difficult to image with molecular resolution using a STM due to their hydrophilicity. Therefore, these experiments were performed with $50 \mathrm{mM}$ 1-decanethiol (C10) ink molecules on unpatterned PDMS stamps displacing preformed AD SAMs. Shown in Figure 3 are $500 \AA \times 500 \AA$ STM images of $\mathbf{C 1 0}$ molecules that had displaced the AD SAM for 0,3 , and $10 \mathrm{~min}$. The resulting two-component SAMs formed ordered lattices of both AD and C10, with the $\mathbf{A D}$ regions having larger lattice spacings and smaller apparent heights. As shown here, the stamping time can be used to control the mean fractional $\mathbf{C 1 0}$ coverage; thus, increasing the stamping duration increases the displacement. Concomitant with this increase in displacement is improvement in the molecular ordering of the $\mathbf{C 1 0}$ lattice. With increasing stamping time, these clusters grew and ordered into larger patches, and ultimately, C10 domains completely displaced the AD SAM for 10 min stamping times. However, the printed SAMs have smaller domains, more defects, and more domain boundaries compared to the corresponding SAMs adsorbed from solution. ${ }^{8}$ On the basis of this molecular picture, one may hypothesize that relative contrasts observed in scanning electron micrographs are based on the following phenomenon. The larger lattice spacings and hence lower packing density of the AD SAM, compared to those of both carboxy-terminated and methyl-terminated alkanethiol SAMs, result in larger secondary electron scattering in the $\mathbf{A D}$ regions compared to the patterned regions, rendering the $A D$ regions the lower intensity areas in scanning electron micrographs.

Microcontact Insertion Printing. As a means to place single molecules or small bundles of molecules in patterns in
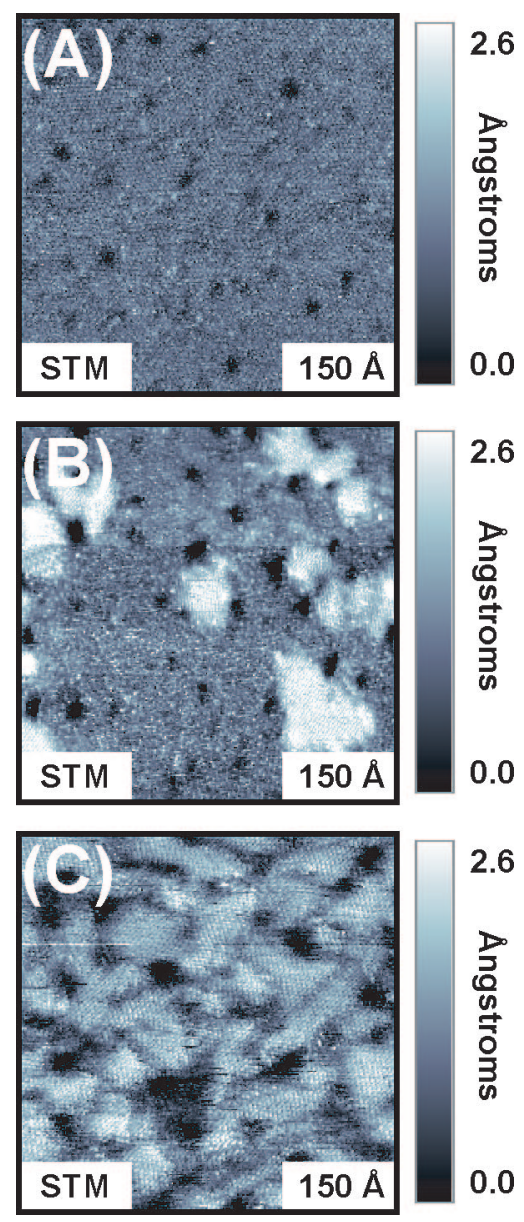

Figure 3. Representative scanning tunneling microscopy images of a two-component, self-assembled monolayer (SAM) of 1-adamantanethiolate (AD) and 1-decanethiolate (C10), fabricated by microdisplacement printing with stamp-substrate contact times of (A) 0 min, (B) 3 min, and (C) $10 \mathrm{~min}$. The concentration of the ink molecule on the unpatterned stamp was $50 \mathrm{mM}$. The ordered lattices of both components can be seen; the less protruding SAM domains (displayed as darker) with the larger lattice spacings are AD. With increasing stamp-substrate contact times, the fractional coverage of C10 increased, displacing the AD and nearly covering the surface for a stamping time of $10 \mathrm{~min}$. The area imaged is $500 \AA \times 500 \AA$, recorded with a sample bias of $-1 \mathrm{~V}$ and tunneling currents of $(\mathrm{A}) 2 \mathrm{pA}$, (B) $1 \mathrm{pA}$, and (C) $2 \mathrm{pA}$.

controlled matrices via soft lithography, $\mu$ CIP utilizes a preassembled alkanethiolate SAM matrix with a selected number and type of defects to facilitate insertion into these defects. The alkanethiolate SAM provides a two-dimensional matrix for the insertion of isolated molecules into film defects, such as structural domain boundaries and substrate step edges, while retaining the molecular ordering of the $n$-alkanethiolate lattice, $^{32}$ and also prevents the lateral diffusion of the inserted molecules.

Shown in Figure 4A is a schematic representation of $\mu$ CIP. Here, a C8 SAM was prepared by deposition in a $1 \mathrm{mM}$ solution of $\mathbf{C} 8$ for $1 \mathrm{~min}$. Then, $10 \mu \mathrm{m} \times$ $10 \mu \mathrm{m}$ posts on a PDMS stamp were inked with $25 \mathrm{mM}$ MUDA and stamped for $30 \mathrm{~min}$. In contrast to the AD 
SAMs in $\mu D P$, the $\mathbf{C 8}$ SAMs have higher intermolecular strengths and smaller lattice spacings, so that they are not displaced; however, isolated ink molecules are inserted into the defects of the C8 SAM via molecular exchange. ${ }^{32-36}$ During $\mu \mathrm{CIP}$, the ink molecules transfer from the stamp and insert into the C8 SAM only in the protruding regions of the PDMS stamp. The patterned substrates were examined with a FESEM (Figure 4B). The resulting regions with inserted molecules, which correspond to the regions with higher intensity in the scanning electron micrographs, did not broaden as observed for $\mu \mathrm{CP}$ patterns for identical ink concentrations and shorter stamping times of the same molecule. $\mu \mathrm{CIP}$ can be repeated to create complex chemical patterns of inserted molecules. This feature is demonstrated in Figure 4C, where a preformed C8 SAM was successively printed with 25 mM MUDA for 30 min each using $25 \mu \mathrm{m} \times 25 \mu \mathrm{m}$ and $10 \mu \mathrm{m} \times 10 \mu \mathrm{m}$ posts on PDMS stamps. The double-patterned regions shown have higher intensities than the single-patterned regions of inserted MUDA molecules. This increase is due to the additional MUDA molecules inserted into the overpatterned regions, which remained in contact with the protruding regions of the elastomeric stamp for twice as long. Furthermore, higher magnification FESEM images showed considerable variation in the uniformity of intensity within the MUDA-inserted regions, indicating the dilute nature of the chemical pattern (data not shown).

To understand the contrast observed in the scanning electron micrographs in Figure 4, to determine the precise locations of insertion, and to quantify the extent of insertion, complementary high-resolution STM studies were performed. The polar nature of the carboxyterminated SAMs serves to enhance the contrast observed in scanning electron micrographs by inducing a larger change in the local surface potential, but it precludes us from obtaining STM images of these molecules with molecular resolution. For this reason, STM studies were performed with methyl-terminated SAMs of similar alkyl chain lengths (C12). Although we attempted to image chemical patterns of C12 molecules inserted into a C8 matrix with the FESEM, we were unable to discern any contrast between the patterned and background regions due to the difference of only four methylene units between $\mathbf{C 1 2}$ and $\mathbf{C 8}$ molecules and the identical exposed terminal funtionality. However, we were able to resolve chemical patterns of $\mathbf{C 1 8}$ molecules inserted into a $\mathbf{C 8}$ matrix due to the larger difference in alkyl chain length. These observations indicate that SEM cannot be applied as a ubiquitous technique to image chemical patterns of inserted molecules and sufficient differences in chain length and/or terminal functionality are required. Figure 5A shows a representative STM image of a C8 SAM formed on a Au\{111\} substrate that shows the absence of protruding molecules and the existence of substrate defects in the initial high-quality SAM. Figure $5 B$
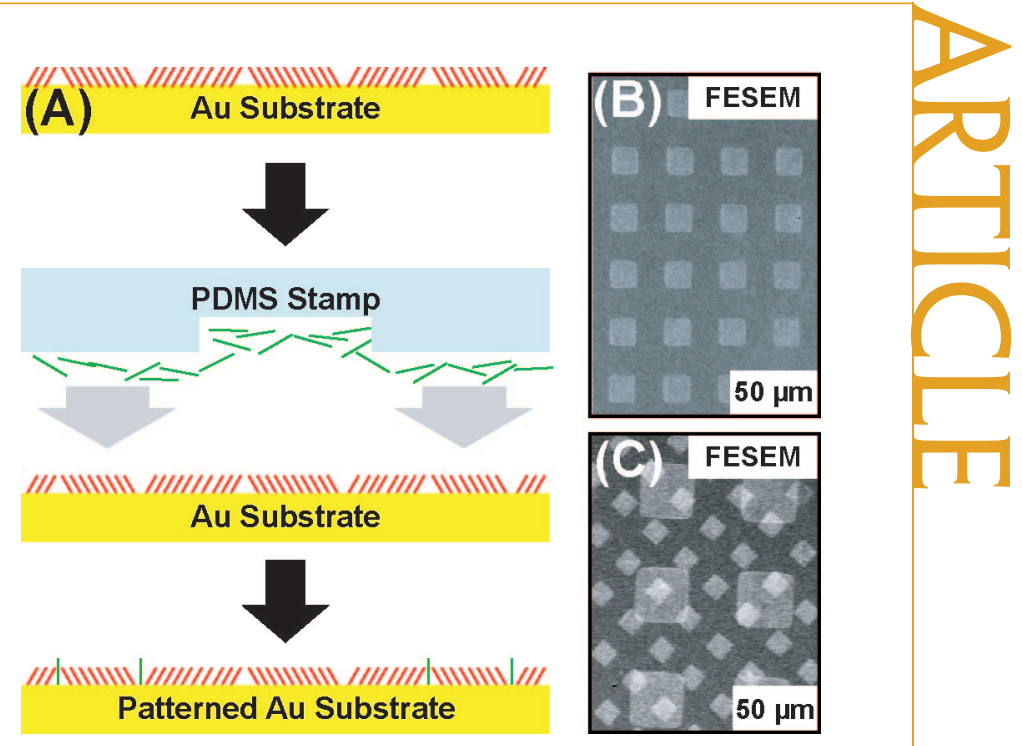

Figure 4. (A) Schematic (not to scale) depicting microcontact insertion printing $(\mu \mathrm{CIP})$ on a 1 -octanethiolate (C8, red lines) self-assembled monolayer (SAM) with a poly(dimethylsiloxane) (PDMS) stamp carrying an ink molecule (green lines). (B) Field emission scanning electron microscope (FESEM) image of a patterned $A u\{111\}$ substrate fabricated by $\mu \mathrm{CIP}$ using $10 \mu \mathrm{m} \times 10 \mu \mathrm{m}$ posts on a PDMS stamp inked with $25 \mathrm{mM}$ 11-mercaptoundecanoic acid (MUDA, $30 \mathrm{~min}$ stamp-substrate contact time). The higher intensity squares correspond to areas where MUDA was inserted, and the low-intensity background corresponds to the unaltered regions of C8 SAM. (C) FESEM image of a patterned Au\{111\} substrate fabricated by double $\mu$ CIP. The substrate was initially patterned with a PDMS stamp with $25 \mu \mathrm{m} \times 25 \mu \mathrm{m}$ posts inked with $25 \mathrm{mM}$ MUDA (30 min stamp-substrate contact time) and then patterned with a PDMS stamp with $10 \mu \mathrm{m} \times 10 \mu \mathrm{m}$ posts (rotated $45^{\circ}$ ) inked with $25 \mathrm{mM}$ MUDA (30 min stampsubstrate contact time). The highest intensity areas appear where the film was inserted with MUDA twice. These micrographs were imaged at a primary electron beam voltage of $1 \mathrm{kV}$ and a collector voltage of $+300 \mathrm{~V}$.

shows a STM image of a sample fabricated by $\mu$ CIP on such a C8 SAM with an unpatterned PDMS stamp coated with $100 \mathrm{mM} \mathrm{C12}$ for $30 \mathrm{~min}$. The more protruding features originating at the substrate defects correspond to C12, and the less protruding lattice corresponds to C8. Similar to solution- and vapor-phase insertion, the $\mathbf{C 1 2}$ molecules were observed to be inserted into the defect sites within the host-SAM matrix, such as substrate vacancy islands and domain boundaries. ${ }^{32-36}$ The fractional C12 coverage for this stamp time and concentration was determined to be $\sim 8 \%$ by performing the following image analyses. ${ }^{9}$ Briefly, several $500 \AA \times 500 \AA$ STM images of samples containing C8 SAMs printed with unpatterned PDMS stamps coated with $\mathbf{C 1 2}$ molecules were acquired across different regions on the substrates. The individual C12 coverage for each STM image was calculated by counting the number of pixels above a threshold intensity, which was set to be the average between the apparent heights of C8 and C12 in the STM image. Substrate defects such as substrate vacancy islands and step edges were excluded from this calculation. The resulting fractional $\mathbf{C 1 2}$ coverages were then averaged across the different images.

The stamping time can be utilized to tune the amount of insertion and hence the extent of dilution of the inserted chemical pattern. ${ }^{9}$ We found that the mean fractional $\mathbf{C} \mathbf{1 2}$ coverage increased monotonically 

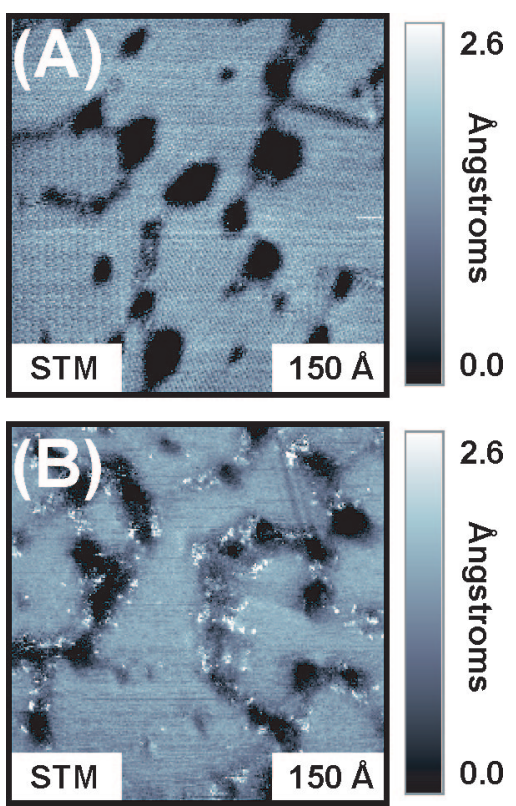

Figure 5. (A) Representative scanning tunneling microscope (STM) image of a 1-octanethiolate (C8) self-assembled monolayer (SAM) formed on a Au\{111\} substrate via exposure to a $1 \mathrm{mM}$ solution of $\mathbf{C 8}$ for $1 \mathrm{~min}$. The most depressed features correspond to substrate vacancy islands. (B) Representative STM image of a two-component SAM of C8 and 1-dodecanethiolate (C12), fabricated by microcontact insertion printing with an unpatterned stamp coated with $100 \mathrm{mM} C 12$ (stamp-substrate contact time of $30 \mathrm{~min}$ ). The area imaged is $500 \AA \times 500 \AA$, recorded with sample biases of -1 and $-1.2 \mathrm{~V}$ and tunneling currents of 2 and $1 \mathrm{pA}$ for (A) and (B), respectively.

with stamping time and saturated at about $\sim 15 \%$ for stamping times of $1 \mathrm{~h}$ and beyond. We expect that this upper limit is imposed by the limited molecular exchange between the C8 SAM and the inserted $\mathbf{C} 12$ molecules, occurring predominantly at defects in the preexisting SAM and not within the crystalline SAM domains on terraces. Using the previously reported timedependent study of the extent of $\mathbf{C 1 2}$ insertion into the C8 SAM, the fractional MUDA coverages for the patterned regions in Figure $4 B, C$ were estimated to be $\sim 8 \%$ for the single-patterned regions on both single $\mu \mathrm{CIP}$ and double $\mu$ CIP regions, and $\sim 16 \%$ for the double-patterned regions of the double $\mu$ CIP sample (assuming that stamping twice for $\mathbf{3 0} \mathrm{min}$ is equivalent to stamping once for $60 \mathrm{~min}$ and that insertion of MUDA is comparable to that of C12). ${ }^{9}$

We note that the SEM provides vastly improved contrast when imaging large areas of low fractional coverages of inserted molecules compared to complementary scans in the LFM (the LFM measures frictional differences across a surface), which highlights the sensitivity of the SEM to small variations in SAM composition. On the basis of the molecular-level understanding derived from the STM images, we infer that, when the ink molecules insert into the defective regions of the host-SAM, the resulting bicomponent SAM has a lower number of defects than the initial SAM. The lower den- sity of defects diminishes the number of secondary electron scattering events in the regions that were contacted by the molecularly inked elastomeric stamp, resulting in increased contrast in the scanning electron micrographs. This phenomenological explanation is consistent with the fact that the regions of doublepatterned $\mu \mathrm{CIP}$ (contacted for twice the stamping time and thus with higher numbers of inserted molecules) appear with higher intensity than the single-patterned $\mu \mathrm{CIP}$ regions. When we imaged chemical patterns of inserted molecules with amine- and methyl-terminated functionality, the regions containing the inserted molecules appeared with higher intensity than the background C8 matrix. Furthermore, we imaged chemical patterns where a MUDA SAM serves as the host matrix and C8 molecules served as the inserted molecules and found that the regions of the inserted molecules still appeared with higher intensity than the background SAM (see Supporting Information, Figure 1, for this data). These observations support the aforementioned argument. Remarkably, chemical patterns of inserted, hydrophilic molecules with dilutions to $<5 \%$ can be distinguished with a SEM (for other inserted molecules, we have found sufficient contrast to observe $0.5 \%$ dilutions). Current work is focused on further derivatizing the isolated molecules with terminal functionalities to serve as small-molecule probes for the detection of biological molecules. ${ }^{37,38}$

Lithography-Assisted Chemical Patterning. In LACP, conventional photolithography is used synergistically with chemical self-assembly and photooxidation of the molecule-substrate bond to create chemical patterns with high fidelity. The central idea is to exploit the ability of a commercially available lift-off resist (LOR 5A, MicroChem Corp., Newton, MA) to withstand conditions necessary for both chemical self-assembly and photooxidation of the molecule-substrate bond (here $\mathrm{Au}-\mathrm{S}$ ) while not disrupting the preexisting SAM. One limitation of soft-lithographic chemical patterning techniques is the inability to register subsequent chemical patterns accurately. Due to the use of photolithography, LACP is capable of creating registered 1:1 chemical patterns over large-area substrates and allows for the feature dimensions to be easily scaled down due to the use of radiation-sensitive polymers for pattern transfer.

The bilayer resist processing used in LACP follows (Figure 6A). A hydrophilic SAM was assembled on a Aucoated Si wafer by immersing the substrate in a $1 \mathrm{mM}$ solution of MHDA in ethanol for $\sim 24 \mathrm{~h}$. A bilayer resist, comprising a lift-off resist (LOR 5A, spin-cast at $4000 \mathrm{rpm}$ and baked for $10 \mathrm{~min}$ at $200^{\circ} \mathrm{C}$ ) and a photoresist (SPR3012, Shipley Co., Marlborough, MA, spincast at $4000 \mathrm{rpm}$ and baked for $60 \mathrm{~s}$ at $95^{\circ} \mathrm{C}$ ), was patterned by a broadband contact aligner $\left(42 \mathrm{~mJ} / \mathrm{cm}^{2}\right.$ at $365 \mathrm{~nm}$ ). After exposure, this resist stack was baked for $60 \mathrm{~s}$ at $115^{\circ} \mathrm{C}$ and developed for $75 \mathrm{~s}$ in a tetramethylammonium hydroxide (TMAH)-based developer (CD- 


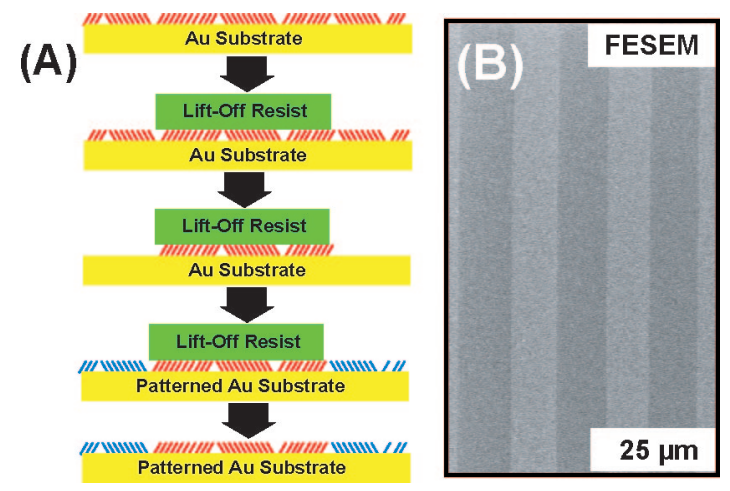

Figure 6. (A) Schematic (not to scale) depicting lithographyassisted chemical patterning of 16-mercaptohexadecanoic acid (MHDA, red lines) and 1-octadecanethiol (C18, blue lines) self-assembled monolayers (SAMs). A robust lithographic resist is patterned atop a MHDA SAM, the pattern is transferred by UV-ozone photooxidation, and a C18 SAM is deposited into the exposed regions. The resist is then removed while maintaining the carboxy-terminal functionality that has been patterned onto the substrate. (B) Field emission scanning electron microscopy image of a patterned two-component SAM comprising MHDA (imaged with lower intensity) and C18. The excellent line-edge quality of the chemical patterns is evident. This micrograph was acquired at a primary electron beam voltage of $1 \mathrm{kV}$ and a collector voltage of $+300 \mathrm{~V}$.

26 , Shipley Co.). While the photoresist serves to pattern the radiation-insensitive lift-off resist, it is unstable in alcohol-based solvents. However, the lift-off resist is chemically robust and withstands self-assembly deposition conditions. ${ }^{39}$ Therefore, the photoresist was selectively removed using acetone while retaining the patterned lift-off resist on the preassembled SAM. The SAM in the exposed patterns of the LOR was removed by UV-ozone oxidation ${ }^{40}$ for 10 min (UV Clean Model 135500, Boekel Scientific, Feasterville, PA) and rinsed in deionized (DI) water and ethanol. The second SAM component was deposited on the substrate from a $1 \mathrm{mM}$ ethanolic solution of $\mathbf{C 1 8}$ for $1 \mathrm{~h}$. Finally, the lift-off resist was removed in an aqueous solution of $2 \% \mathrm{TMAH}$ for $60 \mathrm{~s}$, and the sample was rinsed in DI water and ethanol.

The chemically patterned substrates were imaged with a FESEM (Figure $6 \mathrm{~B}$ ) operating at a primary electron beam voltage of $1 \mathrm{kV}$. The methyl-terminated SAM regions appeared with higher intensity compared to the carboxy-terminated SAM regions. The scanning electron micrographs delineate the excellent line-edge quality of the chemical patterns as a result of the lithographic process utilized in their fabrication. This technique was successfully applied to pattern various combinations of primary (MUDA, MHDA) and secondary, back-filled (C12, C18, MUDA, 11-amino-1undecanethiol (AUDT)) component SAMs, which were imaged with the FESEM with discernable contrast. The ability to pattern two-component SAMs that are carboxy-terminated and amine-terminated exemplifies a unique advantage of this technique; the LOR shields the carboxy-terminated SAM from reacting with the

www.acsnano.org

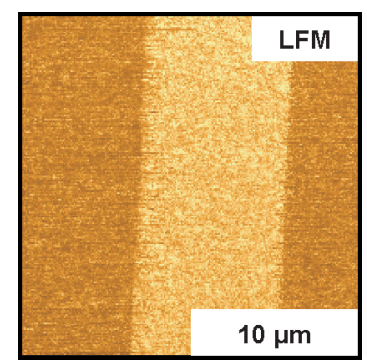

Figure 7. Lateral force microscopy image of patterned two-component self-assembled monolayers comprising 16-mercaptohexadecanoic acid and 1-octadecanethiol. The carboxy-terminated regions have higher friction (shown as lighter) than the methyl-terminated regions. Imaging parameters: force setpoint, $6 \mathrm{nN}$; scan rate, $1 \mathrm{~Hz}$.

amine-terminated SAM. This permits the patterning of high-quality multicomponent SAMs susceptible to undesirable side reactions in other patterning methods. Furthermore, tricomponent SAMs comprising MHDA, AUDT, and C18 were patterned by performing LACP iteratively, enabling the creation of complex chemical patterns that are aligned and have excellent pattern fidelity. ${ }^{10}$

Scanning electon microscopy is a qualitative technique and is incapable of verifying the nature of the terminal functionality of chemical patterns created by LACP. To validate the obtained contrast in scanning electron micrographs further, the chemical patterns were imaged with a LFM. ${ }^{41}$ As shown in Figure 7, the regions of $\mathbf{C} 18$ appeared with lower friction than the regions with MHDA. ${ }^{10,41}$ A priori knowledge of the photomask layout used to create the chemical pattern allowed us to verify that the lower friction regions (C18) matched the regions of higher intensity in the scanning electron micrograph. The regions with $\mathbf{C 1 8}$ have higher secondary electron yields than the regions with MHDA (and hence appear with higher intensity), because the MHDA is more reactive with adventitious adsorbates and more disordered than the $\mathbf{C} 18$ regions. ${ }^{12}$

Contrast Dependence on SEM Operating Voltage. Scanning electon microscopy images of patterned SAMs were first demonstrated by López et al. ${ }^{12}$ and Wollman et al. ${ }^{42}$ López et al. imaged chemical patterns of alkanethiolate molecules patterned by photooxidation of the $\mathrm{Au}-\mathrm{S}$ bond through a stencil mask and by $\mu \mathrm{CP}$.

Wollman et al. modified radiation-sensitive SAMs using a stencil mask and validated the resulting SEM contrast with secondary ion mass spectroscopy. López et al. used the SEM to fabricate and image patterns of proteins adsorbed on SAMs. ${ }^{43}$ In addition, Bittermann et al. studied the effect of molecular packing on the contrast in SEM images and correlated them with scanning force microscopy. ${ }^{13}$ Saito et al. performed $a b$ initio molecular orbital calculations to determine the HOMO and LUMO levels of the adsorbed overlayers and corroborated them with the trends in contrast obtained using the SEM. ${ }^{15}$ Further, Mack et al. attempted to quantify the extent of protein adsorption on pat- 


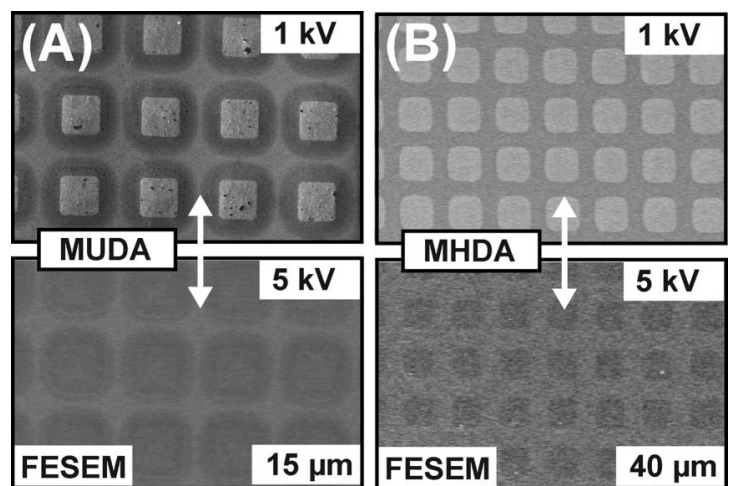

Figure 8. (A) Field emission scanning electron microcopy (FESEM) images acquired at 1 and $5 \mathrm{kV}$ of microcontact printed regions of 11-mercaptoundecanoic acid (MUDA) that mirror the relief pattern on $10 \mu \mathrm{m} \times 10 \mu \mathrm{m}$ posts on a stamp inked with a $25 \mathrm{mM}$ MUDA (10 min stamp-substrate contact time). (B) FESEM images acquired at 1 and $5 \mathrm{kV}$ of microcontact printed regions of 16-mercaptohexadecanoic acid (MHDA) that mirror the relief pattern on $10 \mu \mathrm{m} \times 10 \mu \mathrm{m}$ posts on a stamp inked with a $25 \mathrm{mM}$ MHDA ( $15 \mathrm{~s}$ stamp-substrate contact time). The image contrast interchanged reversibly upon switching back and forth between 1 and $5 \mathrm{kV}$, demonstrating the contrast dependence of self-assembled monolayers on the operating voltage of the SEM.

terned SAMs using the SEM. They found that the normalized SEM intensity increased with increasing alkyl chain length and decreased with increasing thicknesses of adsorbed proteins. ${ }^{14}$ A noticeable aspect of this body of work is the wide range of operating voltages utilized in recording the scanning electron micrographs. A partial list of operating voltages used by the various groups is provided in Table 1.

In each instance, previouly published work attempted to explain the relative contrast observed in the scanning electron micrographs on the basis of the spatial differences in secondary electron scattering and surface potential. Some of the (mutually inclusive) SAM properties considered were the atomic number of the heteroatoms in the functional group, surface potential, surface energy, alkyl chain length, and extent of molec-
TABLE 1. SEM Operating Voltages Used by Different Groups for Imaging SAM Chemical Patterns

research group

López et al. $^{12,43}$

Wollman et al. ${ }^{42}$

SEM operating voltage (kV)

Bittermann et al. ${ }^{13}$

Saito et al. ${ }^{15}$

Mack et al. ${ }^{14}$
3

6

0.6 ular ordering. However, the secondary electron yield $(\delta$, the ratio of the number of secondary electrons to the number of incident primary electrons) is also a function of the primary electron beam energy $\left(E_{\mathrm{PE}}\right)$ used to generate the secondary electrons. ${ }^{44}$ To test if the contrast in the scanning electron micrographs indeed depended on the SEM operating voltage, we imaged $\mu \mathrm{CP}$ patterns of carboxy-terminated molecules. Shown here are $\mu \mathrm{CP}$ patterns of $25 \mathrm{mM}$ MUDA stamped for $10 \mathrm{~min}$ (Figure 8A) and 25 mM MHDA stamped for $15 \mathrm{~s}$ (Figure $8 \mathrm{~B})$. At $1 \mathrm{kV}$, the carboxy-terminated regions had higher secondary electron emission intensity than the bare Au background. However, when imaged at $5 \mathrm{kV}$, the carboxy-terminated regions appeared with lower intensity than the Au background. The contrast continued to switch reproducibly over multiple cycles of moving back and forth between 1 and $5 \mathrm{kV}$, indicating that the contrast reversal was not due to permanent damage to the SAMs. However, similar experiments performed for $\mu \mathrm{DP}$ patterns of MUDA displacing AD did not show contrast reversal with increasing SEM operating voltage (operating voltages up to $25 \mathrm{kV}$ were attempted); the MUDA regions appeared with higher secondary electron emission intensity than the $\mathbf{A D}$ regions at all primary electron beam operating voltages in the range between 0.5 and $25 \mathrm{kV}$ (see Supporting Information, Figure 2, for additional data and explanations).
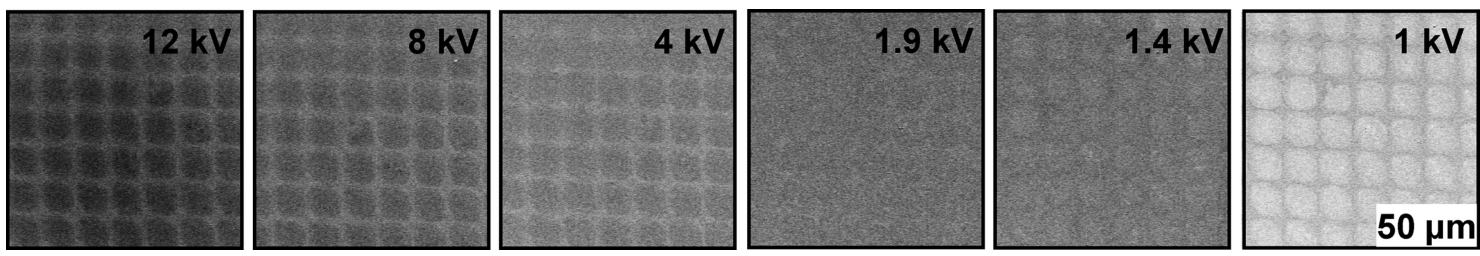

Figure 9. Sequence of field emission scanning electron microscopy images of chemical patterns created by microcontact printing of $25 \mathrm{mM}$ 11-mercaptoundecanoic acid (MUDA, $10 \mathrm{~s}$ stamp-substrate contact time) on Au, recorded over a range of primary electron beam operating voltages. The MUDA regions changed from appearing with lower intensity than Au to appearing with higher intensity than Au at a primary electron beam voltage of $c a .1 .9 \mathrm{kV}$.
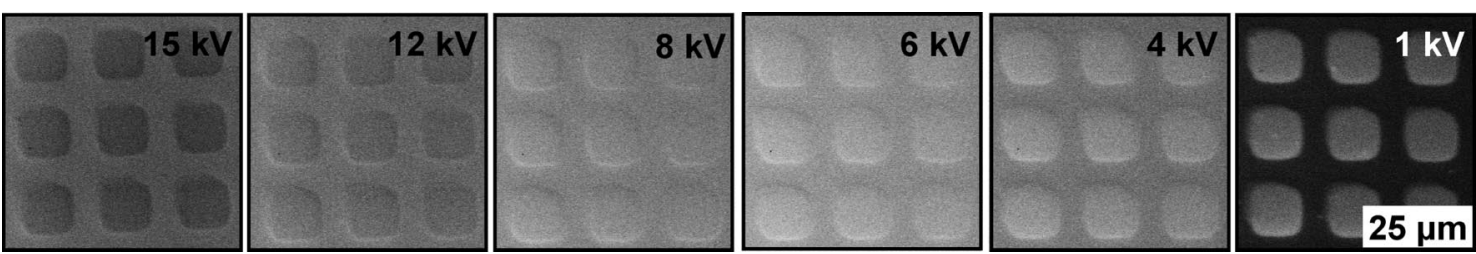

Figure 10. Sequence of field emission scanning electron microscopy images of chemical patterns created by microcontact printing of $25 \mathrm{mM} 11$-mercaptoundecanoic acid (MUDA, $10 \mathrm{~s}$ stamp-substrate contact time) on Au and back-filled with $10 \mathrm{mM}$ 1-dodecanethiol (C12), recorded over a range of primary electron beam operating voltages. The MUDA regions changed from appearing with lower intensity than C12 to appearing with higher intensity than C12 at a primary electron beam voltage of $c a .8 \mathrm{kV}$. 
Based on the semiempirical theory of secondary electron emission, when $\delta$ is plotted as a function of $E_{\mathrm{PE}}$, it follows a bell-shaped curve that is dependent on the material properties (of both the substrate and the organic overlayer). The maximum for this function occurs at $0.7 \mathrm{keV} \leq E_{\mathrm{PE}} \leq 0.9 \mathrm{keV}$ for Au. ${ }^{44}$ Therefore, the imaging conditions in the work described above (Table 1) and here are placed on the high-energy shoulder of the bell-shaped function. The steepness in drop of $\delta$ with increasing $E_{\mathrm{PE}}$ depends on the nature of the SAM. Hence, it may be expected that the contrast seen in the scanning electron micrographs of SAM patterns may interchange with operating voltage if the $\delta$ for the different SAMs and/or Au cross over. In our example, we infer that contrast reversal in $\mu C P$ patterns of MUDA and MHDA is due to the crossover between $\delta_{\text {MUDA }}$ and $\delta_{\text {MHDA }}$ as compared to $\delta_{\mathrm{Au}}$. In the case of the $\mu \mathrm{DP}$ patterns, $\delta_{\text {MUDA }}$ and $\delta_{A D}$ do not cross over; $\delta_{\text {MUDA }}$ is greater than $\delta_{\mathrm{AD}}$ for $E_{\mathrm{PE}}$ up to $25 \mathrm{keV}$.

To test this hypothesis, we collected a series of SEM images of $\mu \mathrm{CP}$ chemical patterns of 25 mM MUDA stamped on Au for $10 \mathrm{~s}$ (Figure 9) and 25 mM MUDA stamped on Au for $10 \mathrm{~s}$ and back-filled with C12 (Figure 10). The $E_{\mathrm{PE}}$ was incrementally varied from 1 to $15 \mathrm{keV}$, and an image was collected at each primary electron beam energy. The collected images were subjected to image analysis where a threshold intensity was used to discern the patterned and bare regions; the average intensity of each region was then determined. Next, the ratios of intensities for patterned to bare regions were calculated and plotted as a function of $E_{\mathrm{PE}}$ (Figure 11).

The sample containing $\mu C P$ patterns of MUDA with a bare Au background showed contrast reversal between the patterned and bare regions on the sample, as the relative contrast ratio between $\delta_{\text {MUDA }}$ and $\delta_{\text {Au }}$ changed from greater than 1 to less than 1 at a primary electron beam voltage of $c a .1 .9 \mathrm{kV}$. The corresponding value for the $\mu$ CP patterns of MUDA/C12 was found to occur at ca. $8 \mathrm{kV}$. This behavior is consistent with the fact that, in $\mu \mathrm{CP}$ patterns of $\mathbf{C} 12$ with a Au background, the $\mathbf{C} 12$ regions yielded higher intensity (and hence higher secondary electron emission) than the background Au regions for the full range of voltages measured. (Note that, at $8 \mathrm{kV}$ for the MUDA/C12 system, there was insufficient contrast in the images to use the automated image analysis routine.) The absolute value of the crossover voltage was found to vary slightly for similarly printed SAMs with different stamping times and concentrations, presumably because of the varying molecular order within the films. These experiments illustrate the strong dependence of the SEM contrast primarily on the operating conditions of the instrument, with additional dependence on the nature of the sample, complicating quantitative analysis.

However, these experiments also show that our understanding of the SEM contrast mechanism in SAM

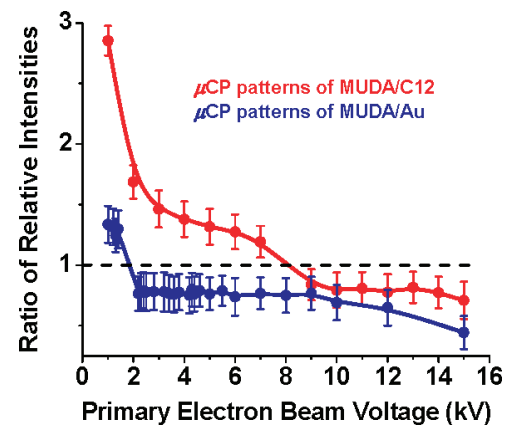

Figure 11. Plot of the relative intensities of microcontact printed ( $\mu \mathrm{CP}$ ) patterns of $25 \mathrm{mM} 11$-mercaptoundecanoic acid (MUDA) stamped for $10 \mathrm{~s}$ on Au (blue) and $\mu \mathrm{CP}$ patterns of 25 mM MUDA stamped for $10 \mathrm{~s}$ on Au and backfilled with 1-dodecanethiol (C12, red), for primary electron beam energies $\left(E_{\mathrm{PE}}\right)$ ranging from 1 to $15 \mathrm{keV}$ (lines are only to guide the eye). The values of secondary electron emission yields $(\boldsymbol{\delta})$ for the different self-assembled monolayer components and $\mathrm{Au}$ vary individually as a function of $E_{\mathrm{PE}}$, with a tendency to decrease with increasing $E_{\mathrm{PE}}$ for the voltage ranges discussed here. Hence, the relative intensities of the different components in a $\mu \mathrm{CP}$ pattern change as a function of $E_{\mathrm{PE}}$. The presence and value of the crossover voltage (i.e., the point at which contrast reversal occurs) depend on the steepness in the drop in $\delta$ vs $E_{\mathrm{PE}}$ for each SAM component and $\mathrm{Au}$. When $\delta$ vs $E_{\mathrm{PE}}$ for the different SAM components and/or $\mathrm{Au}$ intersect and cross over, the relative contrast observed in the scanning electron micrograph reverses. Here $\delta_{\text {MUDA }} / \delta_{\text {Au }}$ changed from values greater than 1 to values less than 1 for $E_{\mathrm{PE}}$ of $c a$. $1.9 \mathrm{keV}$, and the corresponding value for $\delta_{\text {MUDA }} / \delta_{\mathrm{C} 12}$ was ca. $8 \mathrm{keV}$. This plot illustrates the contrast dependence of patterned self-assembled monolayers on the operating voltage of the scanning electron microscope.

patterns is incomplete. Together, these results demonstrate that the SEM can be readily used as a qualitative inspection tool for chemical pattern metrology and is extremely sensitive to compositional variations, even within a molecular monolayer. Thus, the application of the SEM in obtaining chemical information will require the standardization of many parameters, including the energy of the primary electrons, and a better understanding of the complex secondary electron emission and scattering mechanisms of SAMs.

\section{CONCLUSIONS AND PROSPECTS}

Chemical patterns of SAMs show great promise for use in a wide range of applications from electronics to bioanalysis. Microcontact printing is one of the most common procedures to create such chemical patterns; however, it is limited by the tendency of lowermolecular-weight molecules to diffuse laterally across the substrate. Hybrid chemical patterning methods described here enhance many aspects of $\mu \mathrm{CP}$, produce superior pattern fidelity and the ability to control the coverage of patterned molecules, and expand the library of molecules that can be patterned by soft lithography. Furthermore, we have demonstrated the use of the SEM as an analytical tool for the qualitative evaluation of these enhanced chemical patterning methods. In particular, the SEM is capable of distinguishing regions 
of differential mass coverage in patterned SAMs, as well as dilute chemical patterns $(<5 \%)$ of isolated molecules. The current work also delineates the dependence of the contrast in SEM images of SAMs on operating conditions, which points to the as-yet incomplete understanding of the secondary electron emission and scattering mechanisms of molecular overlayers on metal substrates. This indicates the convolution of many parameters, both intrinsic and extrinsic to SAMs, in determining SEM contrast and limits its current application in obtaining quantitative chemical information. However, the SEM is ideally suited to obtain qualitative analytical information such as pattern metrology, with spatial resolution, in chemical patterns of SAMs.

\section{METHODS}

Materials. 1-Octanethiol (C8), 1-decanethiol (C10), 1-dodecanethiol (C12), 1-octadecanethiol (C18, Lancaster, Pelham, NH), 11-mercaptoundecanoic acid (MUDA), 16-mercaptohexadecanoic acid (MHDA, Sigma-Aldrich, Milwaukee, WI), and 11-amino-1-undecanethiol (AUDT, Dojindo Molecular Technologies, Gaithersburg, MD) were purchased and used as received. 1-Adamantanethiol (AD, Sigma-Aldrich) was used as received. ${ }^{45}$

Micrometer-scale relief features on poly(dimethylsiloxane) (PDMS) stamps were generated using $\mathrm{Si} / \mathrm{SiO}_{2}$ masters that were patterned with the desired features using photolithography and reactive-ion etching. ${ }^{45}$ The typical dimensions of the squareshaped raised features on the PDMS stamp were 25,10 , and $5 \mu \mathrm{m}$; each was 1-2 $\mu \mathrm{m}$ high. In preparation for the subsequent stamping step, an ethanolic solution of the ink molecule of appropriate concentration was pipetted directly onto the patterned PDMS stamp, allowed to adsorb on the stamp for $1 \mathrm{~min}$, and then dried with inert gas.

Self-assembled monolayers patterned by $\mu C P, \mu D P$, and $\mu \mathrm{CIP}$ were fabricated on commercially available Au\{111\} evaporated onto freshly cleaved mica substrates (Agilent Technologies AFM, Tempe, AZ), which were annealed using a hydrogen flame just prior to SAM deposition. Patterned SAMs created using LACP were fabricated on Si wafers coated with electron-beamevaporated 10-nm-thick Cr followed by 100-nm-thick Au.

Instrumentation. A LEO 1530 Gemini system (Carl Zeiss, Inc., Oberkochen, Germany) operating at a primary electron beam current of $125 \mathrm{pA}$ at $1 \mathrm{kV}$ (current varied with voltage) was used to collect the FESEM images using an in-lens secondary electron detector. The aperture size was $30 \mu \mathrm{m}$, and the electron detector was maintained at a collection voltage of $+300 \mathrm{~V}$. For acquisition of the micrographs, the electron beam was scanned over the image area for total exposure times less than $20 \mathrm{~s}$ to limit electron-beam-induced damage to the SAMs. ${ }^{46}$ During imaging, the samples were grounded with metallic screws to prevent charge accumulation in the organic regions and the convolution in image contrast thereof. The scanning electron micrographs are shown as obtained; no further image processing was performed.

Scanning tunneling microscopy measurements were performed under ambient conditions using a custom beetle-style STM. ${ }^{47,48}$ Images were recorded in constant-current mode and at high tunneling gap impedances $\left(\sim 10^{12} \Omega\right)$ to ensure large tipsample separation and thus minimal contact between the probe tip and the monolayer. An intensity map accompanies each STM image and corresponds to the apparent height of each pixel in the image. In our images, we represent the more protruding images with higher intensity. Each STM image is representative of images acquired across several regions on a freshly prepared substrate, using a flat/unpatterned stamp, representing the phenomena that occur away from the edges of a micropattern.

Lateral force microscopy images were collected under ambient conditions using a ThermoMicroscopes Autoprobe CP Research AFM (Veeco Instruments, Santa Barbara, CA) in contact mode. The images were recorded at a scan rate of $1 \mathrm{~Hz}$, with a force setpoint of $6 \mathrm{nN}$. Silicon-nitride-coated plank-style AFM tips with a force constant of $0.03 \mathrm{~N} / \mathrm{m}$, or platinum-coated V-style noncontact AFM tips with a force constant of $17.2 \mathrm{~N} / \mathrm{m}$, were used. Both tip styles were purchased from Mikromasch (Portland, OR).
Acknowledgment. This research has been supported by the Penn State Materials Research Institute, Penn State Nanofabrication Facility, Penn State Center for Nanoscale Science (an NSFfunded Materials Research Science and Engineering Center), and the American Chemical Society Division of Analytical Chemistry Fellowship, sponsored by the Society of Analytical Chemists of Pittsburgh (T.J.M.). Parts of this work were conducted at the Penn State node of the NSF-funded National Nanotechnology Infrastructure Network. The authors thank Profs. Susan D. Gillmor and Jennifer R. Hampton and the Penn State Nanofabrication Center staff for helpful interactions and discussions.

Supporting Information Available: Figure 1, FESEM images of patterned Au\{111\} substrates fabricated by $\mu$ CIP using $10 \mu \mathrm{m}$ $\times 10 \mu \mathrm{m}$ posts on PDMS stamps carrying various ink molecules; Figure 2, FESEM images for different chemical patterns at various operating voltages accompanied by representative schematics illustrating the variations of secondary electron yields vs primary electron energy. This material is available free of charge via the Internet at http://pubs.acs.org.

\section{REFERENCES AND NOTES}

1. Kasemo, B. Biological Surface Science. Surf. Sci. 2002, 500, 656-677.

2. Mantooth, B. A.; Weiss, P. S. Fabrication, Assembly, and Characterization of Molecular Electronic Components. Proc. IEEE 2003, 91, 1785-1802.

3. Smith, R. K.; Lewis, P. A.; Weiss, P. S. Patterning SelfAssembled Monolayers. Prog. Surf. Sci. 2004, 75, 1-68.

4. Tirrell, M.; Kokkoli, E.; Biesalski, M. The Role of Surface Science in Bioengineered Materials. Surf. Sci. 2002, 500, 61-83.

5. Xia, Y.; Whitesides, G. M. Soft Lithography. Angew. Chem., Int. Ed. 1998, 37, 550-575.

6. Kim, E.; Xia, Y.; Whitesides, G. M. Polymeric Microstructures Formed by Moulding in Capillaries. Nature 1995, 376, 581-584.

7. Piner, R. D.; Zhu, J.; Xu, F.; Hong, S.; Mirkin, C. A. Dip Pen Nanolithography. Science 1999, 283, 661-663.

8. Dameron, A. A.; Hamptonv, J. R.; Smith, R. K.; Mullen, T. J.; Gillmor, S. D.; Weiss, P. S. Microdisplacement Printing. Nano Lett. 2005, 5, 1834-1837.

9. Mullen, T. J.; Srinivasan, C.; Hohman, J. N.; Gillmor, S. D.; Shuster, M. J.; Horn, M. W.; Andrews, A. M.; Weiss, P. S. Microcontact Insertion Printing. Appl. Phys. Lett. 2007, 90, 063114-1-3.

10. Anderson, M. E.; Srinivasan, C.; Hohman, J. N.; Horn, M. W.; Weiss, P. S. Combining Conventional Lithography with Molecular Self-Assembly for Chemical Patterning. Adv. Mater. 2006, 18, 3258-3260.

11. Delamarche, E.; Schmid, H.; Bietsch, A.; Larsen, N. B.; Rothuizen, H.; Michel, B.; Biebuyck, H. Transport Mechanisms of Alkanethiols during Microcontact Printing on Gold. J. Phys. Chem. B 1998, 102, 3324-3334.

12. López, G.; Biebuyck, H. A.; Whitesides, G. M. Scanning Electron Microscopy Can Form Images of Patterns in SelfAssembled Monolayers. Langmuir 1993, 9, 1513-1516.

13. Bittermann, A. G.; Jacobi, S.; Chi, L. F.; Fuchs, H.; Reichelt, R. Contrast Studies on Organic Monolayers of Different Molecular Packing in FESEM and Their Correlation with SFM Data. Langmuir 2001, 17, 1872-1877. 
14. Mack, N. H.; Dong, R.; Nuzzo, R. G. Quantitative Imaging of Protein Adsorption on Patterned Organic Thin-Film Arrays Using Secondary Electron Emission. J. Am. Chem. Soc. 2006, 128, 7871-7881.

15. Saito, N.; Wu, Y.; Hayashi, K.; Sugimura, H.; Takai, O. Principle in Imaging Contrast in Scanning Electron Microscopy for Binary Microstructures Composed of Organosilane Self-Assembled Monolayers. J. Phys. Chem. B 2003, 107, 664-667.

16. Goldstein, J.; Newbury, D. E.; Joy, D. C.; Lyman, C. E.; Echlin P.; Lifshin, E.; Sawyer, L. C.; Michael, J. R. In Scanning Electron Microscopy and X-Ray Microanalysis; Kluwer Academic/Plenum Publishers: NewYork, 2003; pp 207-209.

17. Joy, D. C. Future of E-Beam Metrology: Obstacles and Opportunities. Proc. SPIE 2002, 4689, 1-10.

18. Lui, C. Y.; Jagota, A.; Lin, Y. Y.; Kramer, E. J. Constraints on Micro-Contact Printing Imposed by Stamp Deformation. Langmuir 2002, 18, 1394-1407.

19. Bohm, I.; Lampert, A.; Buck, M.; Eisert, F.; Grunze, M. A Spectroscopic Study of Thiol Layers Prepared by Contact Printing. Appl. Surf. Sci. 1999, 141, 237-243.

20. Eberhardt, A. S.; Nyquist, R. M.; Parikh, A. N.; Zawodzinski, T.; Swanson, B. I. Defects in Microcontact-Printed and Solution-Grown Self-Assembled Monolayers. Langmuir 1999, 15, 1595-1598.

21. Larsen, N. B.; Biebuyck, H.; Delamarche, E.; Michel, B. Order in Microcontact Printed Self-Assembled Monolayers. J. Am. Chem. Soc. 1997, 119, 3017-3026.

22. Love, J. C.; Estroff, L. A.; Kriebel, J. K.; Nuzzo, R. G.; Whitesides, G. M. Self-Assembled Monolayers of Thiolates on Metals as a Form of Nanotechnology. Chem. Rev. 2005, 105, 1103-1170.

23. Kuhnke, K.; Hoffman, D. M. P.; Wu, X. C.; Bittner, A. M.; Kern, $K$. Chemical Imaging of Interfaces by Sum-Frequency Generation Microscopy: Application to Patterned SelfAssembled Monolayers. Appl. Phys. Lett. 2003, 83, 3830-3833.

24. Stranick, S. J.; Parikh, A. N.; Allara, D. L.; Weiss, P. S. Phase Separation of Mixed-Composition Self-Assembled Monolayers into Nanometer Scale Molecular Domains. J. Phys. Chem. 1994, 98, 11136-11142.

25. Poirier, G. E.; Tarlov, M. J.; Rushmeier, H. E. TwoDimensional Liquid Phase and the $p \times \sqrt{ } 3$ Phase of Alkanethiol Self-Assembled Monolayers on $\mathrm{Au}(111)$. Langmuir 1994, 10, 3383-3386.

26. Love, J. C.; Wolfe, D. B.; Haasch, R.; Chabinyc, M. L.; Paul, K. E.; Whitesides, G. M.; Nuzzo, R. G. Formation and Structure of Self-Assembled Monolayers of Alkanethiolates on Palladium. J. Am. Chem. Soc. 2003, 125, 2597-2609.

27. Dameron, A. A.; Charles, L. F.; Weiss, P. S. Structures and the Displacement of 1-Adamantanethiol Self-Assembled Monolayers on Au\{111\}. J. Am. Chem. Soc. 2005, 127, 86978704.

28. Mullen, T. J.; Dameron, A. A.; Weiss, P. S. Directed Assembly and Separation of Self-Assembled Monolayers via Electrochemical Processing. J. Phys. Chem. B 2007, 110, 14410-14417.

29. Mullen, T. J.; Dameron, A. A.; Saavedra, H. M.; Williams, M. E.; Weiss, P. S. Dynamics of Solution Displacement in 1-Adamantanethiolate Self-Assembled Monolayers. J. Phys. Chem. C 2007, 111, 6740-6746.

30. Dameron, A. A.; Mullen, T. J.; Hengstebeck, R. W.; Saavedra, H. M.; Weiss, P. S. Origins of Displacement in 1-Adamantanethiolate Self-Assembled Monolayers. J. Phys. Chem. C 2007, 111, 6747-6752.

31. Saavedra, H. M.; Barbu, C. M.; Dameron, A. A.; Mullen, T. J.; Crespi, V. H.; Weiss, P. S. 1-Adamantanethiolate Monolayer Displacement Kinetics Follow a Universal Form. J. Am. Chem. Soc. 2007, 129, 10741-10746.

32. Cygan, M. T.; Dunbar, T. D.; Arnold, J. J.; Bumm, L. A.; Shedlock, N. F.; Burgin, T. P.; Jones, L., Il; Allara, D. L.; Tour, J. M.; Weiss, P. S. Insertion, Conductivity, and Structures of Conjugated Organic Oligomers in Self-Assembled Alkanethiol Monolayers on Au\{111\}. J. Am. Chem. Soc 1998, 120, 2721-2732
33. Lewis, P. A.; Donhauser, Z. J.; Mantooth, B. A.; Smith, R. K.; Bumm, L. A.; Kelly, K. F.; Weiss, P. S. Control and Placement of Molecules via Self-Assembly. Nanotechnology 2001, 12, 231-237.

34. Donhauser, Z. J.; Mantooth, B. A.; Kelly, K. F.; Bumm, L. A.; Monnell, J. D.; Stapleton, J. J.; Price, D. W.; Rawlett, A. M.; Allara, D. L.; Tour, J. M.; Weiss, P. S. Conductance Switching in Single Molecules through Conformational Changes. Science 2001, 292, 2303-2307.

35. Donhauser, Z. J.; Mantooth, B. A.; Pearl, T. P.; Kelly, K. F.; Nanayakkara, S. U.; Weiss, P. S. Matrix-Mediated Control of Stochastic Single Molecule Conductance Switching. Jpn. J. Appl. Phys. 2002, 41, 4871-4877.

36. Donhauser, Z. J.; Price, D. W.; Tour, J. M.; Weiss, P. S. Control of Alkanethiolate Monolayer Structure Using Vapor-Phase Annealing. J. Am. Chem. Soc. 2003, 125, 11462-11463.

37. Mullen, T. J.; Dameron, A. A.; Andrews, A. M.; Weiss, P. S. Selecting and Driving Monolayer Structures through Tailored Intermolecular Interactions. Aldrichim. Acta 2007, 40, 21-31.

38. Shuster, M. J.; Vaish, A.; Szapacs, M. E.; Anderson, M. E.; Weiss, P. S.; Andrews, A. M. Biospecific Recognition of Tethered Small Molecules Diluted in Self-Assembled Monolayers. Adv. Mater. 2007, in press.

39. Anderson, M. E.; Tan, L. P.; Mihok, M.; Tanaka, H.; Horn, M. W.; McCarty, G. S.; Weiss, P. S. Photolithographic Structures with Precise Controllable Nanometer-Scale Spacings Created by Molecular Rulers. Adv. Mater. 2006, $18,1020-1022$

40. Huang, J.; Hemminger, J. C. Photooxidation of Thiols in Self-Assembled Monolayers on Gold. J. Am. Chem. Soc. 1993, 115, 3342-3343.

41. Wilbur, J. L.; Biebuyck, H. A.; MacDonald, J. C.; Whitesides, G. M. Scanning Force Microscopies Can Image Patterned Self-Assembled Monolayers. Langmuir 1995, 11, 825-831.

42. Wollman, E. W.; Frisbie, C. D.; Wrighton, M. S. Scanning Electron Microscopy for Imaging Photopatterned SelfAssembled Monolayers on Gold. Langmuir 1993, 9, 15171520.

43. López, G. P.; Biebuyck, H. A.; Harter, R.; Kumar, A.; Whitesides, G. W. Fabrication and Imaging of TwoDimensional Patterns of Proteins Adsorbed on SelfAssembled Monolayers by Scanning Electron Microscopy. J. Am. Chem. Soc. 1993, 115, 10774-10781.

44. Seiler, H. Secondary Electron Emission in the Scanning Electron Microscope. J. Appl. Phys. 1983, 54, R1-R18.

45. Dameron, A. A.; Hampton, J. R.; Gillmor, S. D.; Hohman, J. N.; Weiss, P. S. Enhanced Molecular Patterning via Microdisplacement Printing. J. Vac. Sci. Technol. B 2005, 23, 2929-2932.

46. Seshadri, K.; Froyd, K.; Parikh, A. N.; Allara, D. L.; Lercel, M. J.; Craighead, H. G. Electron-Beam-Induced Damage in Self-Assembled Monolayers. J. Phys. Chem. 1996, 100, 15900-15909.

47. Bumm, L. A.; Arnold, J. J.; Dunbar, T. D.; Allara, D. L.; Weiss, P. S. Electron Transfer through Organic Molecules. J. Phys. Chem. B 1999, 103, 8122-8127.

48. Bumm, L. A.; Arnold, J. J.; Charles, L. F.; Dunbar, T. D.; Allara, D. L.; Weiss, P. S. Directed Self-Assembly to Create Molecular Terraces with Molecularly Sharp Boundaries in Organic Monolayers. J. Am. Chem. Soc. 1996, 121, 8017-8021. 